RECYT

Año 21 / No 32 / 2019 / 48-55

\title{
Encapsulación de antioxidantes del concentrado de Yerba Mate: Influencia de las condiciones de secado
}

\section{Encapsulation of antioxidants from Yerba Mate concentrate: Influence of drying conditions}

\author{
José O. Hermosilla Vera ${ }^{1,2, *}$, Miguel E. Schmalko² \\ 1- Facultad de Ciencias y Tecnología (FaCyT). Universidad Nacional de Itapúa (UNI). Abog. \\ Lorenzo Zacarías №255 c/ Ruta №1. Encarnación, Itapúa, Paraguay. \\ 2- Laboratorio de Yerba Mate. Facultad de Ciencias Exactas, Químicas y Naturales (FCEQyN). Universidad \\ Nacional de Misiones (UNaM). Ruta Nacional Nº12, Km 7,5. Posadas, Misiones, Argentina. \\ * E-mail: joseosvaldohermosilla@gmail.com
}

\section{Resumen}

El objetivo del presente trabajo fue estudiar la influencia de las condiciones de secado de cápsulas de extracto de yerba mate (Ilex paraguariensis St. Hilaire). Las mismas se obtuvieron aplicando la técnica de gelificación iónica y fueron sometidas a tres condiciones de secado: microondas y secado convectivo a 60 y $80^{\circ} \mathrm{C}$. Las isotermas de adsorción obtenidas fueron del tipo sigmoidal y la ecuación de GAB fue la que mejor explicó el comportamiento de los datos experimentales, no encontrándose diferencias estadísticas significativas entre los materiales. Los contenidos de polifenoles totales (CPT) y capacidad antioxidante (CAO) presentaron valores entre 20,12 y 19,68 g EAG/100g ms (masa seca) para CPT, y para la CAO entre 20,47 y 19,45 g EAA/100g ms, no encontrándose diferencias estadísticas significativas. El consumo de $5 \mathrm{~g}$ de cápsulas aportaría el $41 \%$ del requerimiento diario de calcio. La velocidad de rehidratación fue mayor para las cápsulas secadas con microondas.

Palabras clave: Yerba mate; Gelificación iónica; Antioxidante; Encapsulación; Secado

\section{Abstract}

The objective of this work was to study the influence of drying conditions on yerba mate extract capsules (Ilex paraguariensis St. Hilaire). They were obtained by applying the ionic gelation technique and were subjected to three drying conditions: microwave and convective drying at 60 and $80^{\circ} \mathrm{C}$. The sorption isotherms obtained were of the sigmoidal type and the GAB equation was the one that best explained the behavior of the experimental data, and no significant statistical differences were found in the three materials. The contents of total polyphenols (CPT) and antioxidant capacity (CAO) presented values between 20.12 and 19.68 g EAG / 100g DM (dry mass) for CPT, and for the CAO between 20.47 and $19.45 \mathrm{~g}$ EAA / $100 \mathrm{~g} \mathrm{DM}$, no significant statistical differences were found. The consumption of $5 \mathrm{~g}$ of capsules, would contribute $41 \%$ of calcium daily requirement. The rehydration rate was higher for the microwave-dried capsules.

Keywords: Yerba mate; Ionic gelation; Antioxidant; Encapsulation; Drying

\section{Introducción}

En la actualidad, el hombre ha generado cambios en el estilo de vida y hábitos alimentarios. La elección racional de alimentos se basa no sólo en satisfacer el hambre y proporcionar los nutrientes necesarios, sino también en prevenir las enfermedades relacionadas con la nutrición y mejorar el bienestar físico y mental. Los alimentos denominados funcionales constituyen un mercado en alza, siendo uno de los temas más estudiados en los últimos años [1]. Una de las áreas de mayor interés en el desarrollo de estos alimentos, se fundamenta en la posibilidad de modular los sistemas redox y antioxidante del organismo, ya que dichos factores han sido implicados en el desarrollo de numerosas enfermedades crónicas y en el proceso de envejecimiento [2].

La yerba mate (Ilex paraguariensis St. Hilaire), es considerada una fuente rica de antioxidantes. Se han encontrado una gran variedad de compuestos que aportan beneficios para la salud, lo que la hace un material ideal para la extracción de compuestos activos y su posterior aplicación como ingrediente funcional [3], [4].

En sistemas alimentarios, el extracto de yerba mate ha demostrado capacidad de prevenir el deterioro oxidativo de productos con alto contenido graso, mostrando un elevado poder antioxidante inclusive a bajas concentraciones [5], [6]. Sin embargo, su sabor característico limita su incor- 
poración a otros alimentos debido a que puede generar rechazo por parte de los consumidores por modificación del sabor original. Los compuestos fenólicos son los de mayor influencia en la capacidad antioxidante del extracto de yerba mate [7]. Las principales clases de ácidos clorogénicos hallados en la naturaleza son los ácidos cafeoilquínicos (CQA), ácidos dicafeoilquínicos (diCQA), y menos comunes los ácidos feruloilquínicos (FQAs).

El interés en el empleo de yerba mate es aprovechar sus propiedades antioxidantes para el desarrollo de un ingrediente funcional que pueda dar lugar a distintos efectos fisiológicos beneficiosos para el organismo humano, y dar respuestas a las expectativas de los consumidores. En el presente trabajo se propone encapsular los antioxidantes a partir del extracto de yerba mate, en matrices de alginato de calcio facilitando la manipulación, conservación y adición a otros alimentos y de esta manera poder superar los inconvenientes de inestabilidad, disminuir el gusto desagradable, y la vida útil del compuesto [8], [9]. Esto permitirá encontrar nuevos mercados y agregar antioxidantes naturales a los alimentos compatibles para evitar enranciamientos.

El secado es uno de los métodos más utilizados en la conservación de alimentos dado que, al reducir la actividad del agua, se evita el crecimiento de microorganismos. El método de secado más utilizado es el secado convectivo. El mismo se aplica al secado de alimentos utilizando aire a temperaturas bajas y moderadas (menores a $100^{\circ} \mathrm{C}$ ). En los últimos años se han empleado nuevos métodos de secado, como ser el secado con microondas.

El objetivo de este trabajo fue estudiar la influencia de las condiciones de secado de las cápsulas sobre las propiedades fisicoquímicas empleando secado convectivo con aire a dos temperaturas $\left(60\right.$ y $\left.80^{\circ} \mathrm{C}\right)$ y microondas.

\section{Materiales y Métodos}

\section{Preparación de las cápsulas}

Para la obtención de las cápsulas se preparó un extracto acuoso de yerba mate soluble (adquirida del mercado local) al $15 \% \mathrm{p} / \mathrm{v}$ y se mezcló con alginato de sodio al $2 \% \mathrm{p} / \mathrm{v}$. Las cápsulas se obtuvieron mediante gelificación iónica por goteo sobre una solución de $\mathrm{CaCl}_{2}$ al $3 \%$ utilizando una bureta (con apertura de salida de 2,5 $\mathrm{mm}$ ) para regular el paso de la solución y la caída de las gotas en la solución de cloruro de calcio. Esta solución estaba contenida en un vaso de precipitados ubicado a una distancia de $10 \mathrm{~cm}$ debajo del pico de la bureta y era mantenida con agitación magnética. Las cápsulas permanecieron en dicha solución durante $15 \mathrm{~min}$. Luego fueron filtradas y lavadas con agua destilada y posteriormente fueron secadas [4].

\section{Cinética de secado}

Para determinar la cinética las cápsulas fueron secadas en microondas y secado convectivo con aire a 60 y $80^{\circ} \mathrm{C}$. Para la primera se utilizó una potencia 90 watts, pesando cada 5 minutos hasta llegar a peso constante. Posteriormente éstas fueron enfriadas y almacenadas en recipientes herméticos. El secado convectivo se realizó en una capa delgada de las cápsulas colocadas en canastillas. Se pesó la muestra inicial (aproximadamente $100 \mathrm{~g}$ ) y luego fueron llevadas al secadero. En ambos casos se utilizó aire con una humedad absoluta de aproximadamente $0,01 \mathrm{~kg}$ agua/ $\mathrm{kg}$ aire seco y una velocidad de $1 \mathrm{~m} / \mathrm{s}$ en flujo paralelo. Los datos experimentales se obtuvieron durante el transcurso del secado, pesando las muestras en una balanza marca SARTORIUS-WELKE GMBH tipo 2257, industria alemana, con una precisión de $\pm 0,01 \mathrm{~g}$ a intervalos de 15 minutos para $60^{\circ} \mathrm{C}$ y 10 minutos para $80^{\circ} \mathrm{C}$ hasta peso aproximadamente constante. Con estos valores, el de la humedad final y las pesadas previas, se determinó la variación del contenido de humedad en función del tiempo.

\section{Contenido de humedad}

Para determinar el contenido de humedad se utilizó el método de pérdida de masa en estufa a $103^{\circ} \mathrm{C}+/-2^{\circ} \mathrm{C}$ hasta pesada constante (aproximadamente 5 horas). Se determinó el peso de las muestras antes y después de ser llevadas a estufa en una balanza analítica ANR con precisión de $+/-0,0001 \mathrm{~g}$.

\section{Isotermas de adsorción}

Para determinar las isotermas de adsorción se utilizó el método estático. Para ello las cápsulas fueron introducidas, por triplicado, en recipientes cerrados con diferentes soluciones salinas saturadas en equilibrio, las cuales generaban atmósferas de una $\mathrm{a}_{\mathrm{w}}$ determinada. Los recipientes se dispusieron en una estufa a $25^{\circ} \mathrm{C} \pm 1^{\circ} \mathrm{C}$, donde permanecieron hasta alcanzar el equilibrio (aproximadamente 15 días). Luego se determinó el contenido de humedad de las muestras. Las sales utilizadas, así como el valor de $\mathrm{a}_{\mathrm{w}}$ utilizadas se presentan en la Tabla 1.

Tabla 1: Rango de actividades de agua $\left(a_{w}\right)$ de las soluciones salinas saturadas a $25^{\circ} \mathrm{C}$.

\begin{tabular}{|c|c|}
\hline Sales & $\mathrm{a}_{\mathrm{w}}$ \\
\hline $\mathrm{LiCl}$ & 0,113 \\
\hline $\mathrm{MgNO}_{3}$ & 0,528 \\
\hline $\mathrm{CoCl}_{2}$ & 0,649 \\
\hline $\mathrm{NaBr}$ & 0,575 \\
\hline $\mathrm{NaNO}_{3}$ & 0,742 \\
\hline $\mathrm{NaCl}$ & 0,752 \\
\hline $\mathrm{KCl}$ & 0,843 \\
\hline
\end{tabular}


Los datos experimentales de contenido de humedad y $\mathrm{a}_{\mathrm{w}}$ se ajustaron a las ecuaciones de GAB (Guggenheim, Anderson y de Boer), Halsey y Henderson (Tabla 2). La calidad del ajuste de los modelos propuestos se evaluó por medio del coeficiente de correlación lineal $\left(\mathrm{R}^{2}\right)$. Se utilizó el programa Statgraphics Centurion XVII, Statpoint Technologies.

Tabla 2: Ecuaciones utilizadas para el modelado de las isotermas de sorción.

\begin{tabular}{|c|c|c|}
\hline Modelo & Ecuación & Parámetros \\
\hline GAB & $\begin{array}{l}\text { Ecuación } 1 \\
x=\frac{x_{w} c k a_{w}}{\left(1-k a_{w}\right)\left(1-k a_{w}+C k a_{w}\right)}\end{array}$ & $\begin{array}{l}\text { X: humedad del producto } \\
\text { Xm: humedad del producto } \\
\text { cuando los puntos de adsorción } \\
\text { primarios están saturados } \\
\text { por moléculas de agua } \\
\text { C: constante de Guggenheim } \\
\text { relacionada con el calor de } \\
\text { sorción de la monocapa } \\
\text { K: factor de corrección } \\
\text { relacionado con el calor de } \\
\text { sorción de la multicapa }\end{array}$ \\
\hline Halsey & $\begin{array}{c}\text { Ecuación } 2 \\
X=\left(\frac{A}{\ln (1)}\right)^{1 / B}\end{array}$ & $\begin{array}{l}\text { A y B: constantes del modelo } \\
\text { B: caracteriza el tipo } \\
\text { de interacción entre el } \\
\text { vapor y el sólido }\end{array}$ \\
\hline Henderson & $\begin{array}{l}\text { Ecuación } 3 \\
1-a w=e^{n}{ }^{n}\end{array}$ & k y n son constantes \\
\hline
\end{tabular}

\section{Determinación del contenido de polifenoles totales}

Las cápsulas fueron trituradas en un molino de acero inox (marca Arcano), luego se tamizó utilizando una malla de 420 micra. Se pesaron 0,2 $\pm 0,01 \mathrm{~g}$ de muestras tamizadas y se introdujeron en tubos cónicos para luego agregar $5 \mathrm{~mL}$ de metanol al 70\%, la mezcla se homogeneizo en Vortex unos segundos para luego llevarla a un baño termostatizado a $70^{\circ} \mathrm{C}$ durante 5 minutos. Se dejó enfriar y se centrifugó a 2.052 g (3500 rpm) durante 10 minutos. El sobrenadante fue retirado y reservado en un tubo graduado. El proceso de extracción se realizó nuevamente y el sobrenadante obtenido fue combinado con el reservado de la primera extracción. El volumen final de los extractos combinados fue llevado a $10 \mathrm{~mL}$ con metanol al $70 \%$.

El contenido de polifenoles totales (CPT) fue determinado mediante el método de Folin-Ciocalteu. Para el análisis se tomó $1 \mathrm{~mL}$ del extracto y se realizó una dilución (1:100) con agua destilada. De esta solución se tomó 1,0 $\mathrm{mL}$ y se colocó en un tubo de ensayo. Se agregaron 5,0 $\mathrm{mL}$ de la solución del reactivo de Folin-Ciocalteu y luego 4,0 $\mathrm{mL}$ de la solución de carbonato de sodio. Los tubos se agitaron, se taparon y se dejaron reposar durante 60 minutos a temperatura ambiente antes de realizar las lecturas de absorbancia. Como blanco de reactivos se reemplazó 1,0 $\mathrm{mL}$ del extracto diluido con agua destilada. Las lecturas de absorbancia se realizaron a $765 \mathrm{~nm}$ usando una cubeta de cuarzo de $1 \mathrm{~cm}$ de camino óptico. Para obtener la curva de calibrado se prepararon soluciones de ácido gálico en concentraciones de 0 a $50 \mu \mathrm{g} / \mathrm{mL}$. Se realizaron dos determinaciones analíticas por extracto. El contenido de polifenoles totales en el extracto original se expresó como $\mathrm{g}$ de polifenoles totales equivalentes a ácido gálico en 100 $\mathrm{g}$ de materia seca (g EAG \% ms) [10].

\section{Determinación de la capacidad antioxidante}

La capacidad antioxidante (CAO) se determinó mediante el ensayo del radical libre DPPH, usando ácido ascórbico como estándar y se expresó como g equivalentes a ácido ascórbico por cada $100 \mathrm{~g}$ de materia seca (g EAA $\% \mathrm{~ms}$ ). Para ello, $1 \mathrm{~mL}$ de cada extracto de cápsulas de yerba mate obtenido fue diluido (1:25) con agua destilada. Luego, se mezclaron $100 \mu \mathrm{L}$ de cada extracto de yerba mate diluido con $3 \mathrm{~mL}$ de una solución metanólica de DPPH $(100 \mu \mathrm{L} / \mathrm{L})$ en un frasco de color caramelo. Los frascos de reacción se incubaron en estufa a $37^{\circ} \mathrm{C}$ durante $2 \mathrm{~h}$. Las lecturas de absorbancia se realizaron a $517 \mathrm{~nm}$ usando una cubeta de cuarzo de $1 \mathrm{~cm}$ de camino óptico y como blanco se reemplazaron los $100 \mu \mathrm{L}$ de cada extracto diluido por metanol. La curva de calibración preparada con concentraciones de ácido ascórbico entre 0 y $100 \mu \mathrm{g} / \mathrm{L}$ resultó $\mathrm{y}=-0,0421 * \mathrm{x}+1,033\left(\mathrm{R}^{2}=0,9988\right)$, siendo $\mathrm{x}$ la cantidad del estándar $(\mu \mathrm{g})$ utilizada en el ensayo.

\section{Velocidad de rehidratación}

Las cápsulas, contenidas en un tamiz colador, fueron sumergidas en un baño de agua a temperatura ambiente. Se extrajeron del baño en diferentes períodos de tiempo $(5,10$, 20 y 30 minutos), fueron secadas con papel tissue para retirar el agua excedente y luego fueron pesadas. La velocidad de rehidratación se obtuvo dividiendo la ganancia de agua del material rehidratado por el peso del material seco [11] potato, carrot, banana, pepper, garlic, mushroom, onion, leek, pea, corn, pumpkin, and tomato. Las mediciones se realizaron por triplicado para cada muestra. Los valores de ganancia de humedad obtenidos fueron ajustados al modelo matemático de Pilosof [12].

\section{Determinación del contenido calcio}

Para determinar la concentración de Ca se utilizaron $2 \mathrm{~g}$ de cápsulas secas, las que fueron incineradas en una mufla a $525 \pm 25^{\circ} \mathrm{C}$. Al residuo se le agregaron $100 \mathrm{~mL}$ de una solución de $\mathrm{HCl}$ al $10 \%$ y la solución obtenida luego fue filtrada. Se extrajeron $10 \mathrm{~mL}$ y se diluyó con agua en una relación $1 / 10$. Para medir la concentración iónica de $\mathrm{Ca}$, se utilizó un espectrofotómetro de absorción atómica (Marca Analyst 200, Perkin Elmer) y se trabajó a una longitud de onda de $422.7 \mathrm{~nm}$. Se realizaron dos mediciones para cada tipo de cápsulas y se informaron los valores medios [13]. 


\section{Medición de los parámetros de color}

Para la medición del color se utilizaron las cápsulas secadas en las diferentes condiciones. Se colocó una porción de cada muestra en un platillo de vidrio y se determinaron los parámetros de color: luminosidad $\left(\mathrm{L}^{*}\right)$, rojo-verde $\left(\mathrm{a}^{*}\right)$ y amarillo-azul ( $\left.b^{*}\right)$ con un colorímetro modelo EZ4500L Mini Scab de Hunter Lab en tres puntos diferentes. Para el análisis de los datos se utilizaron los valores medios de tres réplicas por muestra.

\section{Resultados y Discusión}

\section{Obtención de Cápsulas}

Se obtuvieron cápsulas de alginato de calcio conteniendo extracto de yerba mate, aplicando la técnica de gelificación iónica. El tamaño de las cápsulas obtenidas fue poco uniforme, debido a que en el mismo influyen varios factores como el efecto de la gravedad, la tensión superficial de la solución que induce la gelificación y el tamaño del pico de la bureta que influyeron en gran medida en la esfericidad y el tamaño de las cápsulas obtenidas. Las cápsulas presentaron pequeños poros en la superficie. De acuerdo con otros trabajos realizados, las cápsulas hechas con alginatos presentan un rango de porosidad comprendida entre 5 a $200 \mathrm{~nm}$. El mismo es un factor importante en la determinación de la liberación del componente bioactivo. La porosidad se puede reducir de manera significativa por secado parcial de las cápsulas o deshidratación completa [14], [15].

\section{Cinética de secado}

Los valores experimentales de contenido de humedad a diferentes tiempos se pueden observar en la Figura 1.

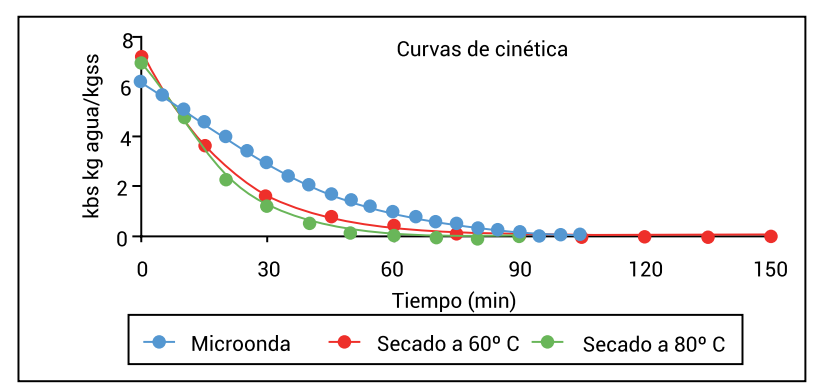

Figura 1: Curvas de cinética de secado de las tres condiciones.

Las cápsulas presentan un comportamiento similar, con una alta velocidad de secado inicial seguida por una etapa más lenta desde aproximadamente los 50 minutos, para llegar finalmente a un tiempo en el que las variaciones del contenido de humedad son muy bajas. En el secado en horno microonda la humedad permanece constante a partir de los 100 minutos, para el secado convectivo a $60{ }^{\circ} \mathrm{C}$ a los 135 minutos, mientras que para el secado convectivo a $80^{\circ} \mathrm{C}$ a los 80 minutos. En el secado convectivo se puede observar que la cinética fue influenciada por la temperatura y que el empleo de temperaturas más elevadas redujo significativamente el tiempo necesario para secar las cápsulas.

En un estudio realizado por Doymaz [16] en el año 2005, se encontró que el efecto de la temperatura fue el más relevante en la disminución del contenido de humedad. De acuerdo con la teoría de la cinética, el aumento de la energía de las moléculas de agua es mayor con la temperatura del aire, generando un escape del vapor de agua de manera fácil y rápida hacia el medio [17]. En cuanto a lo que respecta a la cinética de secado en microonda la temperatura de secado y la potencia son dos de los factores más importantes en el secado por microondas. Estos dos factores tienen influencia significativa sobre los parámetros de secado como con el tiempo de secado [18].

Las cápsulas obtenidas fueron medidas (45 unidades) con un calibre electrónico marca MIYOSHI, encontrándose un diámetro medio igual a $2,99 \mathrm{~mm}$ y una desviación estándar de 0,55 .

\section{Isoterma de adsorción}

Los valores obtenidos de humedad de equilibrio a $25^{\circ} \mathrm{C}$ de las cápsulas de alginato secadas con microonda y en forma convectiva a 60 y $80{ }^{\circ} \mathrm{C}$ fueron ajustados a tres modelos diferentes: GAB, Halsey y Henderson. En la Tabla 3 se presentan los resultados, con sus respectivas constantes y los valores de coeficiente de correlación $\left(\mathrm{R}^{2}\right)$. Se encontró que el modelo de GAB mostró el mejor ajuste a los datos experimentales, con mayores coeficientes de determinación para todos los tratamientos analizadas, en un rango de 0,11 $<$ aw $<0,85$, aplicando un procedimiento de regresión no lineal. En todos los casos se obtuvo un ajuste con $\mathrm{R}^{2} \geq 91 \%$ mientras que en los modelos de Halsey y Henderson se encontraron valores de coeficientes de correlación más bajos. El modelo GAB es uno de los modelos más aceptados para las isotermas de sorción, ya que no solo brinda el valor de humedad de la monocapa $(\mathrm{Xm})$, sino que también se puede conocer las energías de interacción entre la primera capa y las moléculas más lejanas a los sitios individuales de sorción (constantes C y K del modelo) [19].

Los resultados obtenidos en este trabajo con relación al ajuste del modelo de GAB están de acuerdo con los valores límites de las constantes $\mathrm{C}$ y K sugeridos por Lewicki, (1997) [20] basado en el análisis matemático del modelo. Como forma de garantizar una buena descripción de las isotermas del tipo sigmoidal, este autor establece que las constantes deben asumir valores en un rango entre 0,24 $\leq \mathrm{K} \leq 1$ y $5,67 \leq \mathrm{C} \leq \infty$. 
Tabla 3: Parámetros de los modelos matemáticos ajustados para la isoterma de sorción de las cápsulas.

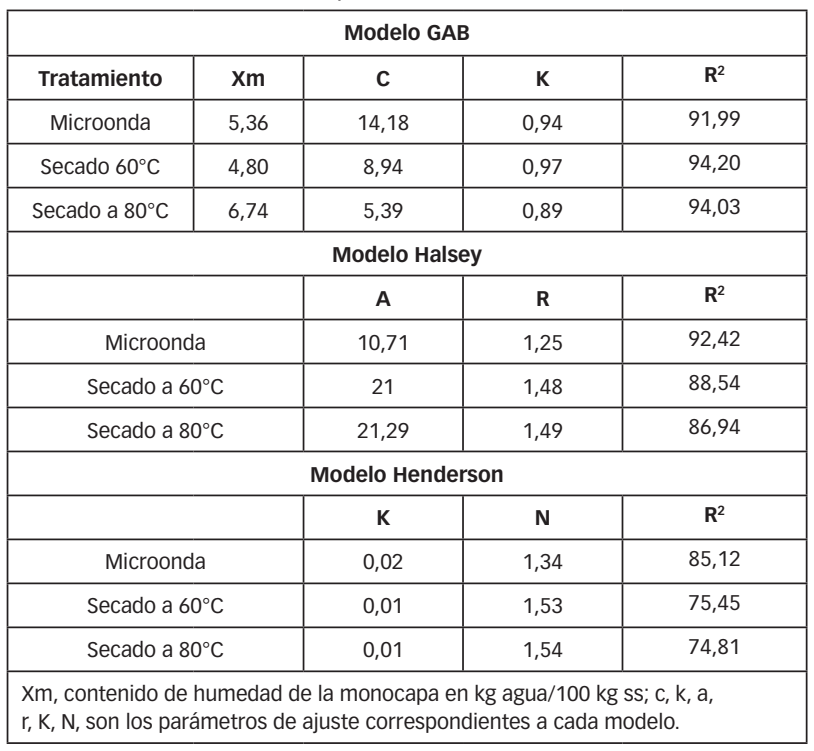

Para las cápsulas con los diferentes tratamientos, los resultados presentan valores de $\mathrm{Xm}$ en el rango entre 4,80 a $6,74 \mathrm{~kg}$ de agua/100kg de solido seco (Tabla 3).
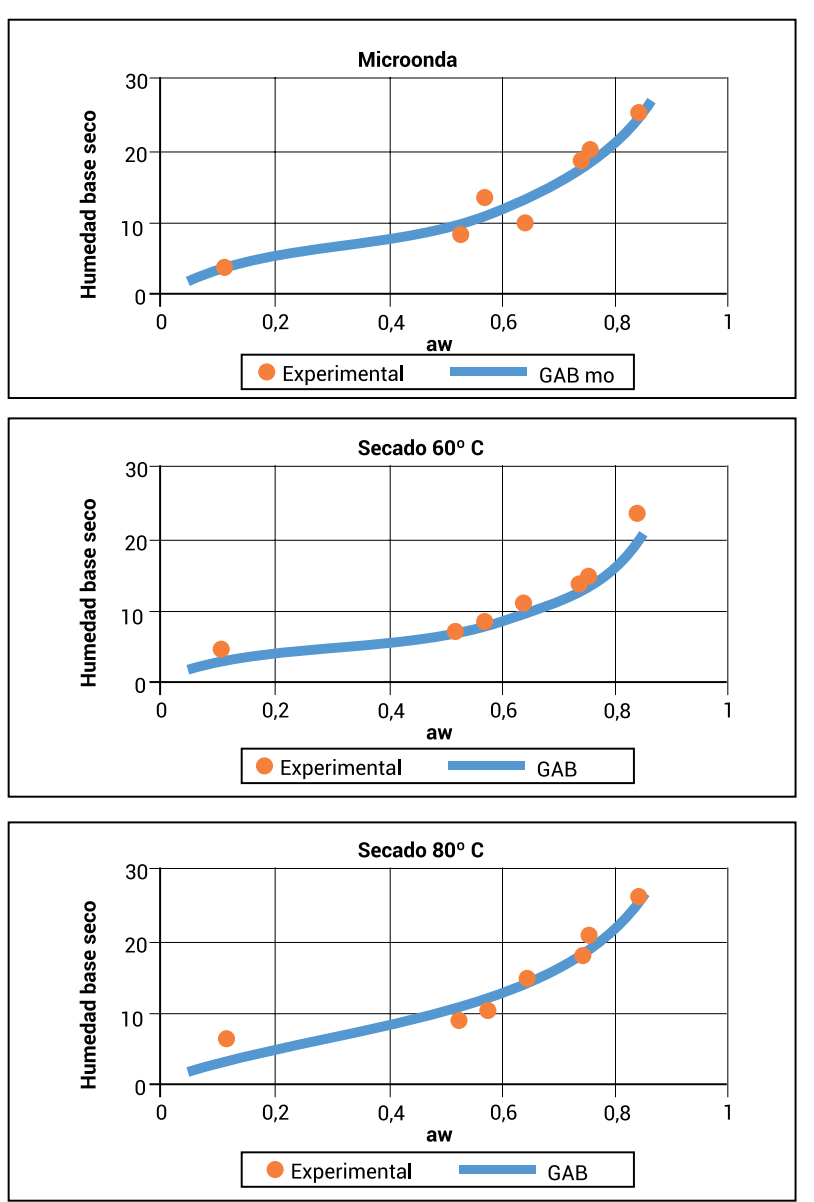

Figura 2: Isotermas de adsorción de las cápsulas. Valores experimentales y predichos con el modelo de GAB.

En la Figura 2 se presentan las curvas de las isotermas de adsorción a $25^{\circ} \mathrm{C}$ de los materiales estudiados. En todos los casos fueron del tipo II, sigmoideas según lo estableci- do por la clasificación de Brunauer [21].

De las curvas de las isotermas se obtuvieron los valores de humedad a los que se pueden almacenar las cápsulas de antioxidantes para que sean microbiológicamente estables a partir de un valor de 0,6 de actividad de agua, encontrándose valores de $11,66 \mathrm{~kg}$ agua $/ \mathrm{kg}$ ss (solido seco) para secado en microonda, $10,36 \mathrm{~kg}$ agua $/ \mathrm{kg}$ ss para secado convectivo a $60^{\circ} \mathrm{C}$ y $12,45 \mathrm{~kg}$ agua/ $\mathrm{kg}$ ss para secado convectivo a $80^{\circ} \mathrm{C}$. En todos los casos el contenido de humedad debe ser inferior a estos valores para almacenar las cápsulas en condiciones seguras.

\section{Contenido de polifenoles y capacidad antioxidante}

Los resultados de contenidos de polifenoles totales y capacidad antioxidantes de las cápsulas sometidas a los tres procesos de secado se presentan en la Tabla 4. En la misma se muestran los valores medios y las desviaciones estándar. Al realizar un análisis estadístico se encuentra que el tipo de secado no ejerce una influencia significativa sobre el contenido de polifenoles y capacidad antioxidantes de las cápsulas. Estos resultados coinciden con los obtenidos en el trabajo realizado por Escalada [22] en el año 2011 donde estudiaron los efectos de la zona de cultivo, tipo de secado y época de cosecha sobre los contenidos de polifenoles totales y sobre la capacidad antioxidante en yerba mate llegando a la conclusión que el tipo de secado no influye en los contenidos de polifenoles.

Tabla 4: Contenido de polifenoles totales y capacidad antioxidante con su desvió estándar.

\begin{tabular}{|c|c|c|}
\hline Tratamiento & CPT (g EAG $\%$ ms) & CAO $($ g EAA \% ms) \\
\hline Microonda & $19,68 \pm 0,81$ & $19,45 \pm 0,80$ \\
\hline Secado convectivo a $60^{\circ} \mathrm{C}$ & $19,93 \pm 0,19$ & $21,93 \pm 0,48$ \\
\hline Secado convectivo a $80^{\circ} \mathrm{C}$ & $20,12 \pm 0,46$ & $20,47 \pm 0,67$ \\
\hline
\end{tabular}

\section{Velocidad de Rehidratación}

En la Tabla 5 se muestran los valores de los parámetros cinéticos y los parámetros de bondad de ajuste de los datos cinéticos de adsorción de agua que se ajustaron al modelo de Pilosof. El ajuste de los datos experimentales, de la adsorción de agua en cápsulas deshidratadas fue bueno, como lo muestran los valores de $\mathrm{R}^{2}$.

Tabla 5: Constantes del modelo de Pilosof para cápsulas secadas en las tres condiciones.

\begin{tabular}{|c|c|c|c|}
\hline Tratamiento & $\begin{array}{c}\mathbf{Q} \\
\text { (kg agua/kg ss) }\end{array}$ & $\begin{array}{c}\text { "b" } \\
\text { (min) }\end{array}$ & $\mathbf{R}^{\mathbf{2}}$ \\
\hline SMO & 2,22 & 1,21 & $98,42 \%$ \\
\hline S60 & 2,35 & 9,13 & $97,06 \%$ \\
\hline S80 & 2,12 & 9,14 & $99,32 \%$ \\
\hline
\end{tabular}

Los valores de Q (valor final de $\mathrm{W}$ ) presentaron valores muy similares en las tres condiciones de secado estudiadas, 
siendo menores a 2,35 $\mathrm{kg}$ de agua $/ \mathrm{kg}$ de sólido seco. En tanto que los valores de b (tiempo necesario para absorber la mitad de la cantidad máxima de agua) se puede notar que en el secado convectivo presentan una relación directa entre ellos variando entre 9,13 a 9,14 minutos, mientras que el secado en microonda presentó un valor mucho menor e igual a 1,21 minutos. En la Figura 3 se pueden observar las curvas de rehidratación en las tres condiciones de secado. Se puede observar que las cápsulas secadas en microonda presentaron ganancia de agua más rápida y con un mayor valor final. Las cápsulas secadas a 60 y $80^{\circ} \mathrm{C}$ tuvieron un comportamiento similar.

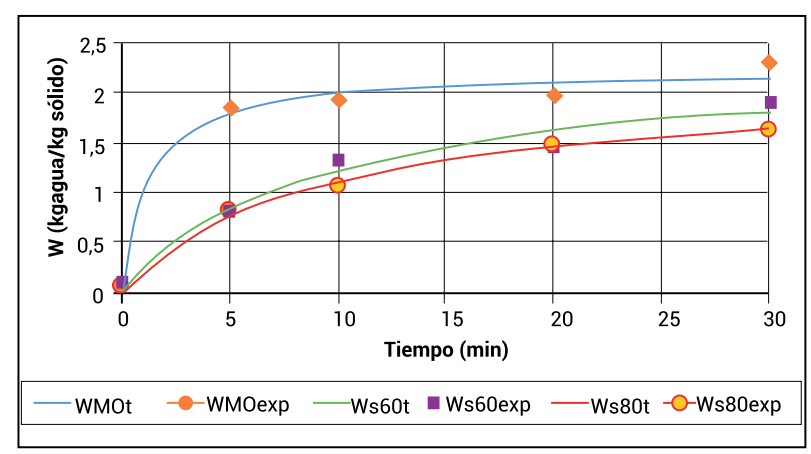

Figura 3: Modelo de Pilosof. Curvas de rehidratación de las cápsulas en las tres condiciones de secado: W: ganancia de agua; MO: microondas; S60: secado convectivo a $60^{\circ} \mathrm{C}$; S80: secado convectivo a $80^{\circ} \mathrm{C}$; subíndices t: teórico y exp: experimental

La intensidad y el mecanismo de deshidratación, sea aire caliente, osmótica, liofilización y microondas, afectan la permeabilidad de las membranas celulares, la formación de estructuras porosas y sumado a una pérdida de sólidos puede ejercer un efecto perjudicial sobre la rehidratación [23].

\section{Determinación del contenido de calcio}

La concentración de este ion depende de la preparación que se realiza y no del método de secado, pues no se modifica con este procesamiento. Las determinaciones se realizaron por duplicado en cada producto y los resultados se pueden observar en la Tabla 6. El límite de detección fue de $0,5 \mathrm{mg} / \mathrm{g}$ y el límite de cuantificación fue de $1 \mathrm{mg} / \mathrm{g}$.

Tabla 6: Contenido de Calcio en las cápsulas en los diferentes productos.

\begin{tabular}{|c|c|c|c|c|}
\hline Tratamiento & $\begin{array}{l}\text { Concentración } \\
\text { (mg/g ss) }\end{array}$ & $\begin{array}{l}\text { Valor } \\
\text { medio }\end{array}$ & $\begin{array}{c}\text { Desviación } \\
\text { standard }\end{array}$ & $\begin{array}{c}\text { Límites de } \\
\text { confianza al } 95 \%\end{array}$ \\
\hline \multirow{2}{*}{ MO } & 134 & \multirow{6}{*}{109} & \multirow{6}{*}{19,6} & \multirow{6}{*}{$88-129$} \\
\hline & 125 & & & \\
\hline \multirow{2}{*}{$\mathrm{SC} 60^{\circ} \mathrm{C}$} & 112 & & & \\
\hline & 108 & & & \\
\hline \multirow{2}{*}{$\mathrm{SC} 80^{\circ} \mathrm{C}$} & 84 & & & \\
\hline & 89 & & & \\
\hline
\end{tabular}

De acuerdo al valor promedio, el consumo de $5 \mathrm{~g}$ por día de estas cápsulas aportaría el $41 \%$ de la dosis diaria requerida de este mineral (1300 mg/día) [24]. Este valor corresponde a la ingesta recomendada que figura en el rotulado nutricional de alimentos. Binaghi y colaboradores [25] encontraron que la biodisponibilidad del calcio no presentó modificaciones cuando se utilizó en preparaciones con yerba mate, utilizándose en leche fortificada.

\section{Color}

En la Figura 4 se muestran las comparaciones de medias e intervalos LSD (nivel de confianza del 95\%) para cada parámetro de color ("L*", "a*" y "b*") de las tres condiciones de secado.
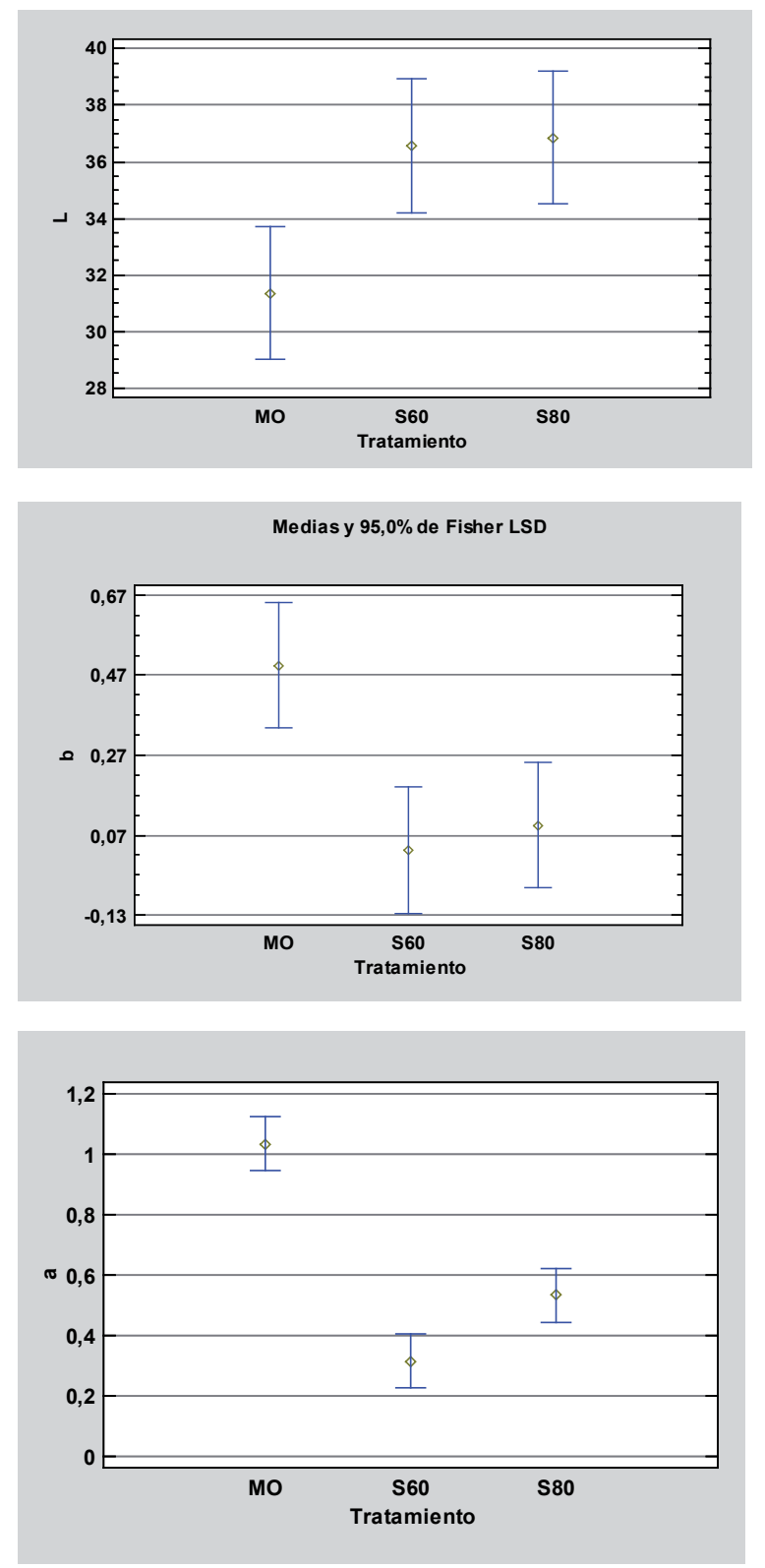

Figura 4: Comparación de los parámetros de color " $\mathrm{L}^{\star}$ ", "a*" $\mathrm{y}$ " $\mathrm{b}$ " entre las cápsulas secadas en distintas condiciones.

Respecto a los parámetros "L*", “a*” y "b*" se puede mencionar que las cápsulas secadas a 60 y $80^{\circ} \mathrm{C}$ no mues- 
tran variaciones estadísticamente significativas entre ellas mientras que, en el secado con microonda, se observa que muestran valores significativamente mayores en los parámetros "a*" y "b*", y un menor valor de " $L$ *". Esto significa que las cápsulas tratadas en microonda presentaron una tonalidad marrón más clara respecto al secado convectivo, pudiendo esta diferencia deberse a la elevada velocidad de calentamiento que no provoca cambios significativos en la superficie del alimento cuando se trabaja con tiempos y potencias de microondas adecuados [26] el secado se llevó a cabo mediante la deshidratación osmótica y luego a través del secado combinado (microondas y convección con aire caliente.

\section{Conclusiones}

Al encapsular el extracto de yerba mate en una matriz de alginato de calcio, las cápsulas obtenidas presentaron forma esférica irregular y pequeñas porosidades en las superficies. Al determinar las curvas de cinéticas de secado de las cápsulas se observó que en el secado convectivo influyó la temperatura del aire de secado mientras que el tiempo final de secado con microonda fue intermedio al de las dos temperaturas de secado convectivo. Las isotermas de adsorción de las cápsulas, determinadas a $25^{\circ} \mathrm{C}$, presentaron formas sigmoideas tipo II y el modelo de GAB mostró el mejor ajuste en el modelado de los datos experimentales en las tres condiciones de secado. Los contenidos de polifenoles presentaron valores entre 20,12 y 19,68 g EAG/100g ms y la capacidad antioxidante entre 20,47 y 19,45 g EAA/100g ms, no se encontraron diferencias significativas entre los diferentes métodos de secado. Se encontró que el consumo de $5 \mathrm{~g}$ de cápsulas aportaría 41\% de la ingesta diaria recomendada de calcio, encontrándose que el método de secado no influye sobre el mismo. Las cápsulas secadas con microonda presentaron un color marrón más claro y mayor velocidad de rehidratación respecto al secado convectivo. Las cápsulas de extractos de yerba mate obtenidas representan una alternativa para uso como ingrediente funcional pudiéndose incorporar a alimentos (sopas, yogures, otros preparados lácteos) y productos deshidratados que se venden como mezclas (mix de frutas, turrones, etc.).

\section{Agradecimientos}

Al Consejo Nacional de Ciencias y Tecnología (CONACYT) del Paraguay, por la beca otorgada para la realización de esta investigación, también a la Facultad de Ciencias y Tecnología de la Universidad Nacional de Itapúa y Facultad de Ciencias Exactas, Químicas y Naturales de la Universidad Nacional de Misiones por las instalaciones y los equipos otorgados.

\section{Bibliografía}

1. Falguera, V.; Aliguer, N. y Falguera, M. An integrated approach to current trends in food consumption: Moving toward functional and organic products?, Food Control. 26, No 2, p. 274-281. 2012.

2. Wootton-Beard, P.C. y Ryan, L. Improving public health?: The role of antioxidant-rich fruit and vegetable beverages., Food Res. Int. 44, No 10: p. 3135-3148. 2011.

3. Deladino, L.; Anbinder, P.S.; Navarro, A.S. y Martino, M.N. Encapsulation of natural antioxidants extracted from Ilex paraguariensis., Carbohydr. Polym. 71, No 1: p. 126-134. 2008.

4. López, A.F.; Deladino, L.; Navarro, A.S. y Martino, M.N. Encapsulación de compuestos bioactivos con alginatos para la industria de alimentos., Limentech Cienc. y Tecnol. Aliment. 10, No 1: p. 18-27. 2012.

5. De Campos, R.M.L.; Hierro, E.; Ordóñez, J.A.; Bertol, T.M.; Terra, N.N. y de la Hoz, L. Fatty acid and volatile compounds from salami manufactured with yerba mate (Ilex paraguariensis) extract and pork back fat and meat from pigs fed on diets with partial replacement of maize with rice bran., Food Chem. 103, No 4: p. 1159-1167. 2007.

6. Valerga, J.; Reta, M. y Cecilia, M., LwT. Food Science and Technology Polyphenol input to the antioxidant activity of yerba mate (Ilex paraguariensis) extracts., LWT - Food Sci. Technol. 45, No 1: p. 28-35. 2012.

7. Butiuk, A.P.; Martos, M.A.; Adachi, O. y Hours, R.S. Study of the chlorogenic acid content in yerba mate (Ilex paraguariensis St. Hil.): Effect of plant fraction, processing step and harvesting season., J. Appl. Res. Med. Aromat. Plants. 3, $\mathrm{N}^{\mathrm{o}}$ 1: p. 27-33. 2016.

8. Fang, Z. y Bhandari, в. Encapsulation of polyphenols - A review. Trends Food Sci. Technol. 21, No 10: p. 510-523. 2010.

9. Munin, A. and F. Edwards-Lévy. Encapsulation of natural polyphenolic compounds; a review. Pharmaceutics. $3, \mathrm{~N}^{\mathrm{o}}$ 4: p. 793-829. 2011.

10. Thea, M.E.; Bohaczenko, E. A.; Schmalko,M.E. "Variación del contenido de polifenoles totales y la capacidad antioxidante de la yerba mate según diferentes tipos de estacionamiento," Actas del XIV Congreso CYTAL de Ciencia y Tecnología de los Alimentos. Rosario, Argentina, pp. 1-6, 2012.

11. Krokida, M.K. y Marinos-Kouris, D. Rehydration kinetics of dehydrated products., J. Food Eng. 57, No 1: p. 1-7. 2003.

12. Pilosof Boquet, A.R. y Bartholoma, G. Kinetics of Water Uptake by Food Powders. FOOD Sci. 50, No 7985: p. 278-279. 1985.

13. Schmalko, M.E.; Acuña, M.G. y Scipioni, G.P. The Use of Maltodextrin Matrices to Control the Release of Minerals from Fortied Mate. International Journal of Food Studies.1: p. 17-25. 2012.

14. Fundueanu, G.; Nastruzzi, C.; Carpov, A.; Desbrieres, J. y Rinaudo, 
M. Physico-chemical characterization of Ca-alginate microparticles produced with different methods., Biomaterials. 20, No 15: p. 1427-1435. 1999.

15. George, M. y Abraham, T.E. Polyionic hydrocolloids for the intestinal delivery of protein drugs: Alginate and chitosan - a review., J. Control. Release. 114, $\mathrm{N}^{\mathrm{o}} 1$ : p. 1-14. 2006.

16. Doymaz, I. Drying characteristics and kinetics of okra., J. Food Eng. 69, № 3: p. 275-279. 2005.

17. Rodriguez, A. Ósmosis Directa-Microondas y Secado Convectivo Por Aire Caliente-Microondas Para La Deshidratación De Frutos Del Bosque., Tesis doctoral en ingeneria. Universidad Nacional de La Plata. 2014.

18. Correa Cuevas, L.P. y Hernández Baltazar, E. El uso de las microondas en la industria farmacéutica., Rev. Mex. Ciencias For. 13, No 2: p. 112. 2012.

19. Rubiano, H.J.; Cárdenas, K. y velásquez, J. Evaluación de las propiedades termodinámicas y térmicas del d-limoneno encapsulado., Rev. U.D.C.A Actual. Divulg. Científica. 18, $\mathrm{N}^{\circ}$ 2: p. 425-434. 2015.

20. Lewicki, P. The applicability of the GAB model to food water sorption isotherms., Int. J. food Sci. Technol. 32, No 1945: p. 553-557. 1997.

21. Brunauer, W.E.D. y Deming, L.S. "On a Theory of the van der Waals Adsorption of Gases", Journal of the American Chemical Society, vol. 62, No 6, p. 1723, 1940.

22. Escalada, G.; Brumovsky, L.A. y Hartwig, v.G. Influencia de la zona de cultivo y procesamiento de la yerba mate sobre su contenido de polifenoles totales y capacidad antioxidante. Cienc. y Tecnol. No 15: p. 66-74. 2011.
23. García-mogollón, c. y Romero-barragán, A. A. Capacidad de Rehidratación y Cambio de Color de Yuca (Manihot esculenta crantz) Deshidratada en Microondas Rehydration Capacity and Color Change of Cassava (Manihot esculenta crantz) Dried in Microwave. Inf. tecnol. 27, $\mathrm{N}^{\mathrm{o}} 1$ : p. 53-60. 2016.

24. Cabo Masip, T.; Alentado Morell, N. y Dalmau Serra, J. Nuevas recomendaciones diarias de ingesta de calcio y vitamina D: Prevención del raquitismo nutricional., Acta Pediatr. Esp. 66, No 5: p. 233-236. 2008.

25. Binaghi, M.J.; Pellegrino, N.R. y Valencia, M.E. Bioaccesibilidad de minerales en infusiones de yerba mate (Ilex paraguariensis St.) y en mezclas con leche fortificadas con hierro., Archivos Latinoamericanos de nutrición. 61 (1): p- 81-86. 2011.

26. Della Rocca, P. Secado de alimentos por métodos combinados: Deshidratación osmótica y secado por microondas y aire caliente., Tesis de maestria en tecnologia de alimentos. Universidad Tecnológica Nacional. 2010 .

Recibido: 29/08/2018.

Aprobado: 22/02/2019. 